\title{
Mesures et analyses de l'écoulement dans une roue de pompe mixte
}

\author{
Experimental and theoretical analysis \\ of flow in a mixed flow impeller
}

J.M. Duchemin,

Société Métraflu

64, chemin des Mouilles

69130 Ecully
I. Trebinjac,

Laboratoire de mécanique des fluides

Etude centrale lyonnaise

69131 Ecully

Mémoire présenté aux Journées S.F.M./S.H.F. sur la mécanique des fluides, Paris, 9-10 octobre 1985.

Des mesures du champ d'écoulement à l'aval d'une roue de pompe mixte (hélico-centrifuge) ont été effectuées dans l'eau à l'aide d'une sonde cinq trous et d'un vélocimètre laser. Une méthode simple de prédiction des pertes mesurées a été recherchée. Elle se base sur le schéma semi-empirique bien connu établi pour les compresseurs axiaux. Dans le cas considéré, il était de plus nécessaire d'évaluer l'effet de la rotation et de la courbure sur le développement de la couche limite; pour cela, l'approche de Balje a été suivie. Les résultats de l'application de la méthode à une roue mixte de conception différente dont les essais ont été effectués à l'air sont également donnés.

Experimental investigation of the flow downstream of a mixed-flow pump impeller has been made (in water), with a five-hole probe and a laser doppler velocimeter. An attempt to predict measured losses with a simple method based on classical axial flow compressor knowledge has been made. Furthermore, because of the pump's geometry, it was necessary to evaluate curvature and rotation effects on boundary layer growth. The Balje approach has been used for this case. Comparisons between theoretical and experimental results of another mixed flow impeller tested in air are added.

\section{Introduction}

Un programme d'investigations expérimentales devant comporter, d'une part, des mesures du champ hydrodynamique à l'amont et à l'aval d'une roue de pompe mixte (helico-centrifuge) avec couvercle et, d'autre part, des mesures de vitesses à l'intérieur de la même roue sans couvercle, est en cours. Pour le présent, seule la première partie a été réalisée.

Bien que ne disposant donc pas encore de mesures internes détaillées du type de celles fournies par Fraser [1], il a semblé intéressant de rechercher un modèle quantitatif simple rendant compte des résultats expérimentaux et de leur enseignement majeur pour le concepteur : le niveau de pertes. 


\section{Notations :}

a

B

$B^{* *}$

$c$

$C_{m}$

$C_{0}, C_{v}$

$D_{\text {eq }}$

$H_{12}$ $i$

$i^{*}$

l

$\vec{N}$

$\mathrm{N}$
$\mathrm{Pa}$

$\mathrm{Pa}^{*}$

r

$R^{*}$

$R$

$R_{m}$

$R_{o r}$

$R_{0 \omega}$

$R_{p}$

$U$

W

$\alpha$

$\beta$

$\eta$

$\varphi$

$\omega$

$\sigma$

$\theta$

Indices

2

$\max$

e

$+$ distance normale entre deux aubes

blocage géométrique

blocage basé sur l'épaisseur de quantité de mouvement

corde

composante méridienne de la vitesse absolue

composante tangentielle de la vitesse absolue

facteur de diffusion équivalent

facteur de forme

incidence

incidence optimale

distance normale entre le bord de fuite et le plan de sondage

normale à l'aube

puissance sur l'arbre

débit réduit

rayon

rayon réduit

rayon de courbure de l'aubage

rayon moyen du bord de fuite

nombre de courbure

nombre de rotation

rayon de courbure de la ligne de courant projetée dans le plan défini par le vecteur vitesse $\vec{W}$ et la normale à l'aube $\vec{N}$

vitesse d'entraînement

vitesse relative

coefficient empirique

angle de la ligne de courant relative avec le plan méridien

rendement

angle entre la normale au bord de fuite et la vitesse méridienne

vitesse angulaire

solidité

épaisseur de quantité de mouvement

relatif au bord d'attaque

relatif au bord de fuite

valeur maximale sur l'aubage

relatif à l'écoulement sain

relatif à l'écoulement mélangé
Calcul des caractéristiques des couches limites au bord de fuite à partir du champ hydrodynamique aval

Nous supposons qu'au bord de fuite l'écoulement peut se décomposer en une zone saine, oủ les composantes de vitesse sont constantes en azimut, et une couche limite bi-dimensionnelle contenue dans le plan déterminé par la vitesse extérieure et la normale à l'aube (fig. l).

$\mathrm{Si}$ nous appliquons les équations intégrales de la dynamique et de continuité au volume ABCDEF (fig. 2) et si nous supposons que le mélange a lieu sur une distance négligeable, il vient :

$$
\begin{aligned}
\eta=1 & -\frac{1}{2} \frac{W_{+}^{2}}{U \cdot C_{\theta}^{+}} \cos ^{2} \beta^{+} \cdot \cos ^{2} \varphi^{+} \\
& \left\{\frac{2 B^{* *}+\left(B+H_{12} B^{* *}\right)^{2}}{\left(1-B-H_{12} B^{* * *}\right)^{2}}+\right. \\
& \left(\operatorname{tg}^{2} \beta^{+}+\operatorname{tg}^{2} \varphi^{+}+\operatorname{tg}^{2} \beta^{+} \cdot \operatorname{tg}^{2} \varphi^{+}\right) \\
& {\left.\left[\left(\frac{1-B-B^{* *} H_{12}}{1-B-B^{* *}\left(1+H_{12}\right)}\right)^{2}-1\right]\right\} }
\end{aligned}
$$

Les détails de la dérivation sont donnés dans la référence [2].

\section{Calcul de la décélération}

La décélération sur l'aubage a été évaluée à partir de la formulation du facteur de diffusion équivalent proposée par Klapproth dans la discussion de [3].

$$
\begin{aligned}
& D_{\mathrm{cq}}=\frac{W_{\max }}{W_{2}}= \\
& \frac{W_{1}}{W_{2}}\left[1,12+\alpha\left(i-i^{*}\right)^{1,43}+0,61 \frac{C_{m l}^{2}}{W_{1}^{2}} \frac{1}{\sigma} K\right] \\
& K=\frac{\left(U-C_{\theta}\right)_{1}}{C m_{1}}-\frac{r_{2}}{r_{1}} \frac{C m_{2}}{C m_{1}} \frac{\left(U-C_{\theta}\right)_{2}}{C m_{2}} \\
& \quad-\frac{\omega r_{1}}{C m_{1}}\left(1-\frac{r_{2}^{2}}{r_{1}^{2}}\right) .
\end{aligned}
$$

La précision du calcul de la décélération par l'emploi de cette méthode semi-empirique est discutée par la suite.

\section{Prédiction de l'état de la couche limite au bord de fuite}

Pour prédire l'état de la couche limite au bord de fuite, il est possible de recourir à des méthodes intégrales ou différentielles, mais les modèles prenant en compte des effets de courbure et de rotation élevés sont encore bien imparfaits.

Une autre approche proposée par Balje [4] consiste à relier de manière semi-empirique l'épaisseur de quantité de mouvement $(\theta / c)_{0+c}$ induite par la décélération avec effets de courbure et de rotation à la quantité de mouve- 


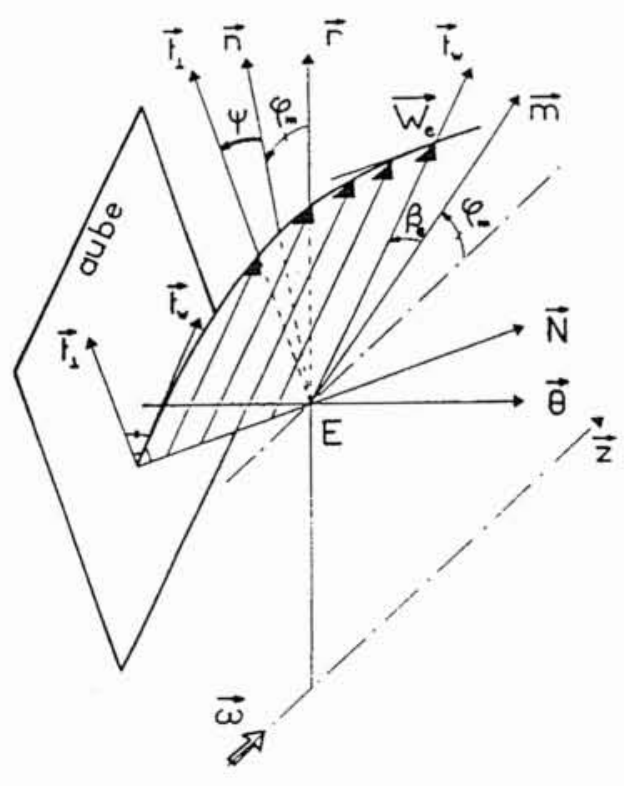

Figure 1. - Couche limite d'aubage.

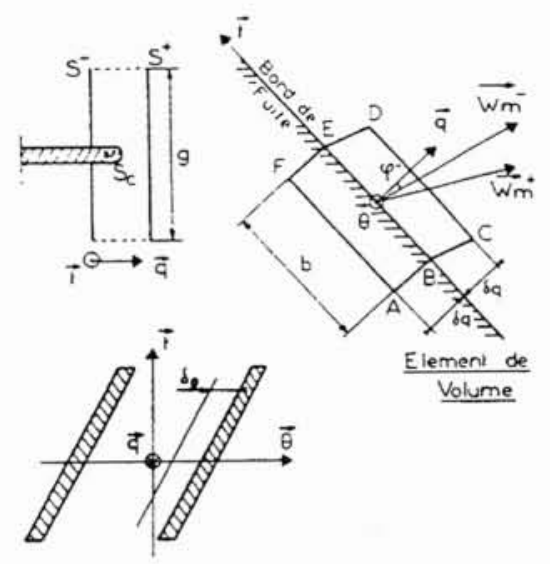

Figure 2. - Elément de volume.

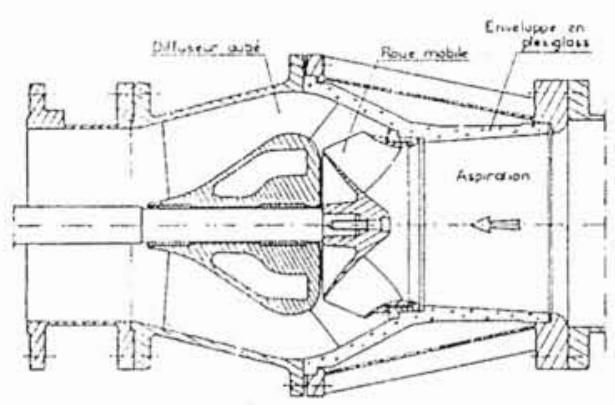

Figure 3. - Coupe méridienne de la pompe.

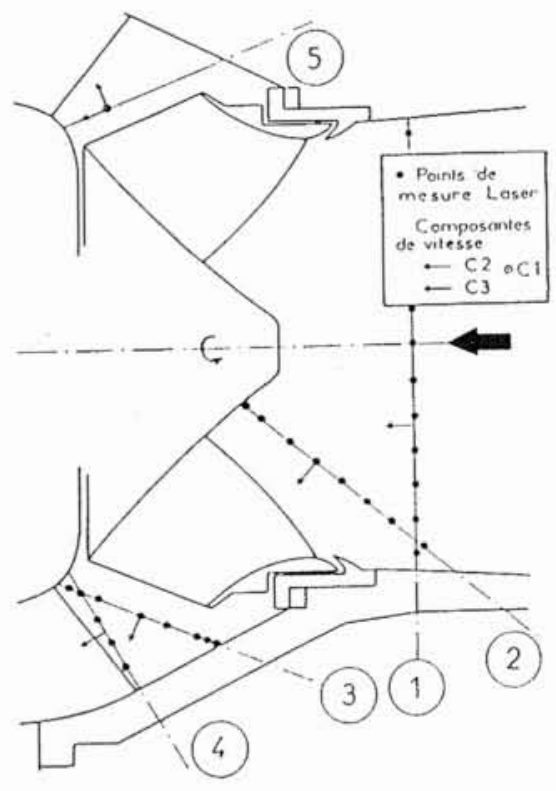

Figure 4. - Sections de mesure. ment $(\theta / c)_{0}$ induite par la même décélération sans courbure ni rotation.

Les deux quantités sont liées par la relation:

$$
\left(\frac{\theta}{c}\right)_{c+\omega}=\left(\frac{\theta}{c}\right)_{0}\left[1+1,5\left(R_{a_{\omega}}+R_{a_{c}}\right)^{1 / 2}\right],
$$

avec :

$$
\begin{aligned}
& R_{0 \omega}=+2 a \omega / W, \\
& R_{0 c}=\frac{a}{R_{c}} .
\end{aligned}
$$

\section{Étude expérimentale}

La roue analysée (roue 1) est une roue de pompe mixte (fig. 3) à aubes couchées en arrière. Le carter est équipé de hublots pour permettre les mesures par vélocimétrie laser. Les essais ont été réalisés à l'eau. Des relevés de pression totale et de vitesse stationnaires ont été effectués à l'aval de la roue à une distance $V / R_{m} 10 \%$ (section 5 , fig. 4) à l'aide d'une sonde directionnelle à 5 prises de pression, à différents débits. D'autre part, une campagne de mesures a été réalisée par vélocimétrie laser à effet Doppler (mode différentiel) à une seule composante, aux sections 1, 2, 3 et 4 . Deux composantes de la vitesse locale et instantanée ont été mesurées successivement, par rotation de l'ensemble optique. 


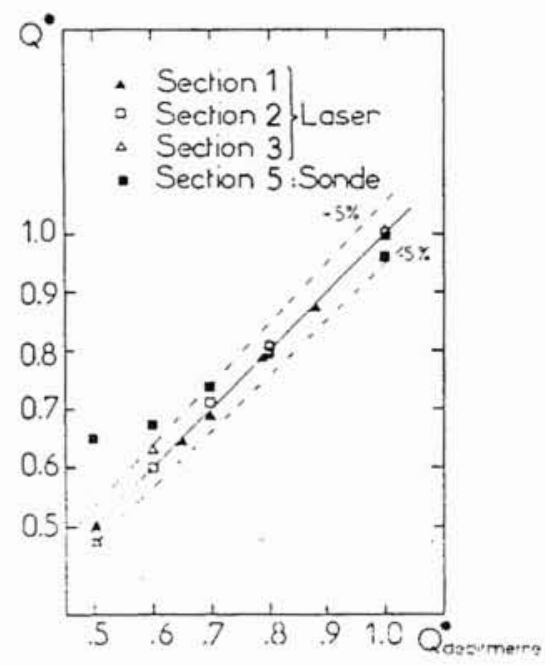

Figure 5. - Comparaison des débits intégré et mesuré.

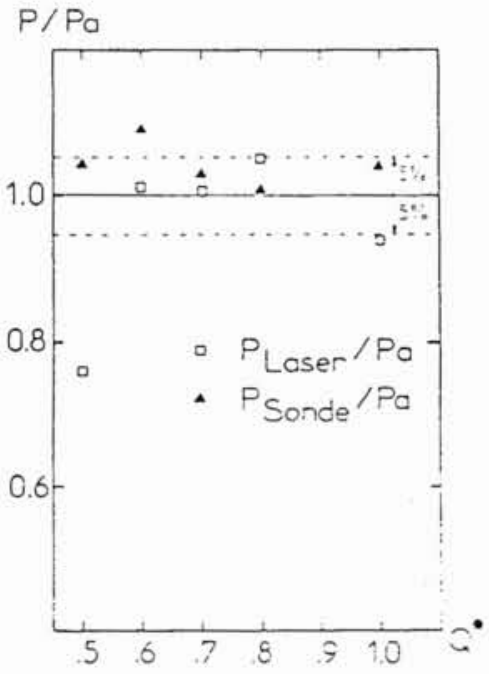

Figure 6. - Comparaison des puissances intégrées et mesurées.

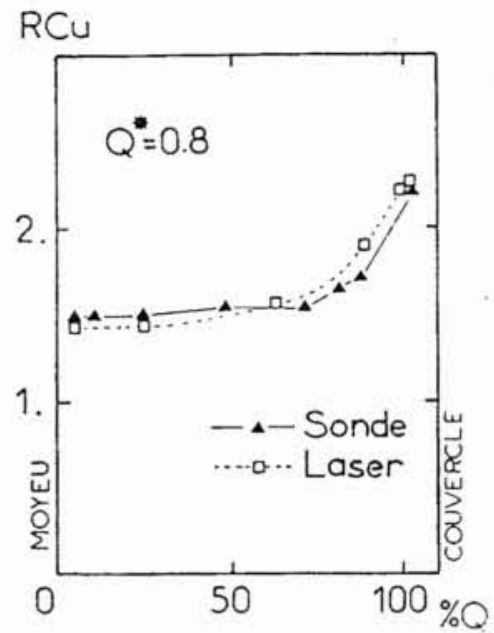

Figure 7. - Comparaison des $\left(\mathrm{rC}_{w}\right)$ en fonction du débit intégré à partir du moyeu.
L'ensemble des résultats a permis, d'une part, de montrer la bonne concordance des deux techniques de mesure (fig. 5, 6 et 7) et, d'autre part, de réaliser une reconstitution cohérente de l'écoulement. Pour plus de détails, on se reportera à la référence [5].

\section{Application de la modélisation aux résultats d'essais}

L'application de la modélisation a été effectuée pour les débits de $0,75 Q_{\mathrm{op}}, Q_{\mathrm{opt}}$ et $1,25 Q_{\mathrm{op}}$. A partir des relevés effectués à la sonde de pression, nous avons évalué les grandeurs hydrodynamiques de l'écoulement mélangé au bord de fuite, les mesures laser nous ayant montré que le mélange était pratiquement réalisé dans les sections de mesure.

Nous avons évalué la décélération sur l'aubage à l'aide de la formule (2), en supposant que l'incidence était voisine de l'incidence optimale $\left(i=i^{*}\right)$.

La précision de cette formule pour notre cas a été testée à l'aide de calculs inter-aubes par la méthode de Katsanis [6] et l'accord sur les valeurs de la vitesse maximale est satisfaisant à environ $6 \%$ en moyenne.

Le calcul des épaisseurs de quantité de mouvement des couches limites au bord de fuite a été effectué à partir de l'expression (1) pour une plage de facteur de forme de 1,4 à 2,4 . Une détermination unique de l'épaisseur de quantité de mouvement est cependant donnée à partir des travaux de Davis [7] qui propose une corrélation de $H_{12}$ avec la décélération. Il est à noter de plus qu'une incertitude importante sur $H_{12}$ entraîne une erreur acceptable sur la valeur $\theta$. A titre d'exemple, quand $H_{12}$ varie de 1,4 à 2,4 la variation de $\theta / c$ est de l'ordre de $15 \%$.

Les valeurs de $\theta / c$ en fonction de $D_{\text {sy }}$ sont portées figure 8 .

D'une manière générale, les valeurs de $\theta / c$ obtenues sont triples de celles en grille axiale. Les causes de pertes supplémentaires sont nombreuses, mais étant donné la valeur élevée de $R_{\phi_{0}}$ nous avons attribué la différence aux effets de la rotation. Ces effets ont été évalués par la corrélation de Balje. Notre type de roue étant géométriquement complexe, les nombres $R_{\phi_{c}}$ et $R_{\phi_{0}}$ ont été recalculés puisqu'il convient de prendre :

- pour les effets de courbure

$$
R_{0 c}=\frac{a}{R_{p}},
$$

- pour les effets de rotation

$$
R_{a_{\omega}}=-2 a(\vec{\omega} \wedge \vec{W}) \cdot \vec{N} / W^{2} .
$$

Les valeurs $(\theta / c)_{0}$ issues de cette correction sont montrées figure 9. La même analyse a été effectuée à l'aide de résultats d'essais réalisés à l'air sur une roue mixte de conception différente. Les valeurs ainsi obtenues sont portées sur cette même figure. 


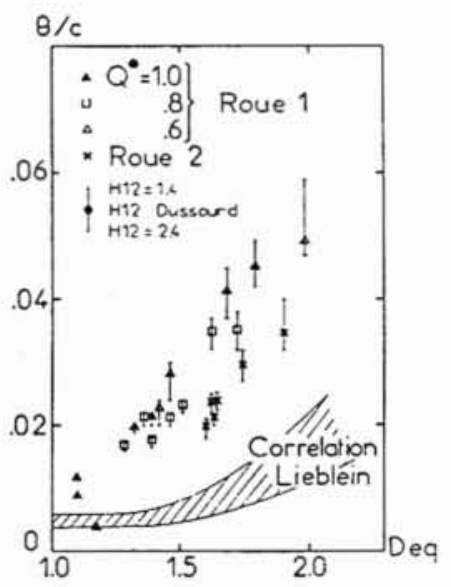

Figure 8. $-(\theta / C)=f\left(D_{\text {eq }}\right)$ $(\theta / c)$.

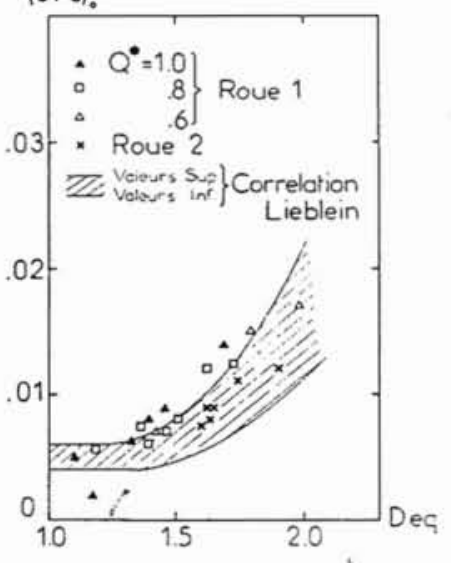

Figure 9. $-(\theta / C)=f\left(D_{\text {eq }}\right)$.

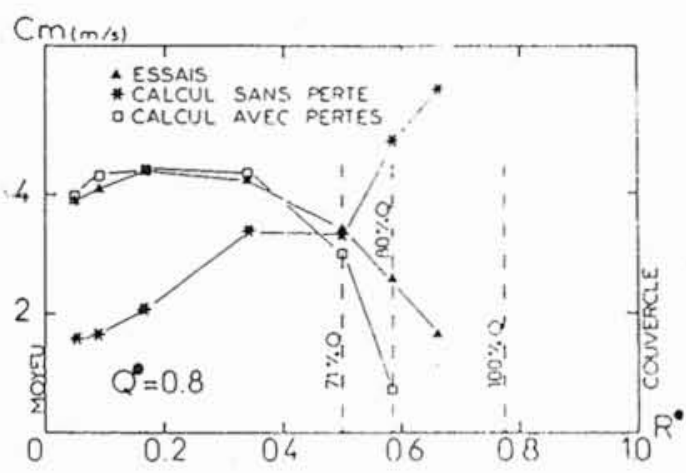

Figure 10. - Distributions de vitesses méridiennes.

\section{Discussion}

L'écart entre les pertes mesurées et les pertes attendues d'un schéma bidimensionnel a été considérablement réduit. Il est à noter que la dispersion des résultats expérimentaux est équivalente à celle obtenue par Lieblein [3] à l'aide d'essais en grilles axiales dans lesquelles la bidimensionnalité de l'écoulement était soigneusement vérifiée.

On remarque cependant qu'une prédiction à partir de ces schémas bidimensionnels conduirait à une légère sous-estimation. Des causes probables de pertes sont ignorées dans notre modèle. A ce propos, on peut faire les remarques suivantes :

- l'écoulement en sortie de roue n'est pas axisymétrique et est fortement rotationnel dans le sillage, comme le montre Fraser [1], ce qui vient augmenter les pertes par mélange,

- dans les zones à proximité du couvercle, les pertes secondaires et les pertes par élargissement brusque viennent s'ajouter aux pertes de profil.

- nous avons négligé le terme d'incidence dans le calcul de $D_{\mathrm{eq}}$, les valeurs en sont donc sous-estimées.

L'équation de la dynamique pour un écoulement axisymétrique (donc totalement mélangé) avec prise en compte des termes de pertes, projetée le long de la direction (5) (voir figure 4), a permis de calculer des répartitions de vitesse méridiennes.

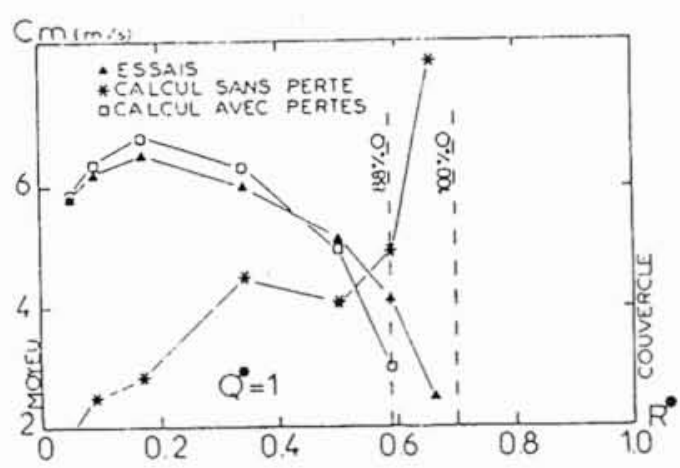

Figure 11. - Distributions de vitesses méridiennes.

Les résultats expérimentaux et des calculs avec prise en compte des pertes expérimentales sont portés sur les figures 10 et 11 . La comparaison avec un calcul sans pertes montre que la prise en compte de celles-ci est indispensable pour restituer la distribution des vitesses méridiennes. 


\section{Conclusion}

L'ensemble de la modélisation pourrait être vérifié sur d'autres roues, d'une manière simple à mettre en œuvre, c'est-à-dire par des mesures à la sonde directionnelle en aval. Il serait toutefois souhaitable que des mesures détaillées, telles que celles présentées dans la référence [1] visant à tester des méthodes numériques tridimensionnelles puissent également servir à valider chaque étape de la modélisation qui, par son aspect global, peut présenter un intérêt certain, notamment dans le domaine de la conception. Moyennant ces vérifications, l'utilisation des corrélations établies en grilles axiales, en tenant compte des effets de la rotation et de la courbure des aubages, paraît être une méthode de prédiction des pertes de profil dans une machine mixte, avec un degré de précision équivalent à celui obtenu lors des prédictions dans un compresseur axial.

Un modèle de calcul des pertes est indispensable pour une bonne prédiction des distributions des vitesses méridiennes et des angles à l'aval de la roue.

\section{Remerciements}

Nous remercions la Société Bergeron qui a mis à notre disposition une de ses pompes, et le ministère de la Recherche et de la Technologie pour son aide financière.

\section{Références}

[1] FRASER S.M., CAREY C., WILSON G. - Behavior of air in the rotor of a model mixed-flow pump operating at peak efficiency, Journal of Fluids Engineering, 107, 183-189 (1985).

[2] DUCHEMIN J.M., TREBINJAC I. - Ecoulement inter-aube à débit partiel dans une roue de pompe hélico-centrifuge. Compte rendu de fin d'études d'une recherche financée par le M.I.R. Contrat D.G.R.S.T. $81 . S .0880$.

[3] LIEBLEIN S. - Loss and stall analysis of compressor cascades, Journal of Basic Engineering, 387-400 (1959).

[4] BALJE O.E. - A flow model for centrifugal compressor rotors, Journal of Engineering for Power, 100, 148-158 (1978).

[5] TREBINJAC I. - Contribution théorique et expérimentale à l'analyse de l'écoulement dans une pompe mixte (hélicocentrifuge), thèse D.I., novembre 1985.

[6] KATSANIS T., NALLY M., WILLIAM D. - Revised Fortran program for calculating velocities and streamlines on a blade-to-blade stream surface of a turbomachane, NASA TM X-1964 (1969).

[7] DAVIS R.C., DUSSOURD J.L. - A unified procedure for the calculation of off-design performance of radial turbomachinery, Journal of Engineering for Power, p. 133-146 (1971). 